\title{
UNA REVISIÓN TEÓRICA SOBRE EL ESTRÉS Y ALGUNOS ASPECTOS RELEVANTES DE ÉSTE EN EL ÁMBITO EDUCATIVO
}

\author{
María Luisa Naranjo Pereira \\ Docente de la Escuela de Orientación y Educación Especial \\ Universidad de Costa Rica, San José, Costa Rica
}

Recibido 3-VIII-2009 • Aceptado 28-VIII-2009 • Corregido 11-X-2009

\begin{abstract}
Resumen: El objetivo de este artículo es comentar los aportes que sobre el tema de estrés presentan varios teóricos y enfoques: fisiológicos, psicosociales, cognitivos e integradores. Se presentan las diferencias entre distrés y eustrés y la importancia de lograr un nivel óptimo de estrés. Se revisan las fases que lo caracterizan: la alarma, la resistencia y el agotamiento y su relación con la ansiedad y la depresión. Se estudian diversas respuestas que pueden manifestarse en la persona, producto de él: físicas, psicosomáticas, cognitivas, emotivas y conductuales. Se analizan algunas de las principales causas generadoras de estrés, tanto aquellas provenientes de situaciones externas como las producidas por las propias características de personalidad y las formas particulares de enfrentar las situaciones del diario vivir. Se revisan aspectos importantes que favorecen la prevención de este, tales como las actitudes, el ejercicio físico, el tipo de alimentación, el empleo del tiempo libre y la planificación personal, así como aquellos que permiten afrontarlo de una mejor manera, entre estos la relajación, la meditación y el papel central de las cogniciones. Finalmente, se relaciona el tema del estrés con la educación, haciendo énfasis en las principales fuentes generadoras de este en la población estudiantil y sus efectos tanto personales como en el rendimiento académico.
\end{abstract}

Palabras clave: Estrés, prevención, afrontamiento, educación.

\section{Estrés}

El estrés ha sido un tema de interés y preocupación para diversos científicos de la conducta humana, por cuanto sus efectos inciden tanto en la salud física y mental, como en el rendimiento laboral y académico de la persona. Provoca preocupación y angustia y puede conducir a trastornos personales, desórdenes familiares e incluso sociales. Al respecto señalan Martínez y Díaz (2007): "el problema que subyace radica en los requerimientos de la Modernidad, concentrada en la obtención de resultados al margen de sus consecuencias sobre la calidad de vida, y por ende en la salud física y mental de las personas afectadas" (p. 1).

Todas las personas necesitan aprender a prevenir y controlar el estrés. Quien no lo hace, puede poner en peligro su salud y su tranquilidad, mientras que quien conoce y pone en práctica acciones adecuadas para prevenirlo y afrontarlo puede disfrutar de un estilo de vida más sano y más satisfactorio.

Autores como Oblitas (2004) se refieren a diferentes concepciones teóricas 


\begin{abstract}
This article aims to discuss the contributions on the topic of stress presented by several theorists and approaches: physiologists, psychosocial, cognitive and integrators. The differences between distress and eustress are analyzed and the importance to achieve an optimum level of stress. Phases that characterize it are analyzed: alarm, resistance and depletion and its relationship with anxiety and depression. In addition, different responses of the person to stress are explored: physical, psychosomatic, cognitive, emotional and behavioral. Some of the main generating causes of the stress are discussed: external situations such as those produced by the own personality characteristics and the particular forms to address situations of daily living. Important aspects that help prevention from it are reviewed, such as attitudes, exercise, the type of food, employment of leisure and personal planning, as well as those that allow facing it better, among this relaxation, meditation and the central role of the cognitions. Finally, relationship between of stress and education is reviewed, emphasizing the main generating sources of it in the student population and its effects in personal life and in academic performance.
\end{abstract}

Key words: Stress, prevention, coping, education. y como estas entienden el término estrés. Este autor explica que los enfoques fisiológicos y bioquímicos se centran en las respuestas orgánicas que se generan en la persona cuando se enfrenta a una situación percibida como amenazante. Por su parte, los enfoques psicosociales hacen énfasis en factores externos; es decir, en los estímulos y eventos productores de estrés. Por su lado, los enfoques con una orientación cognitiva subrayan que el estrés surge a partir de la evaluación cognitiva que la persona realiza tomando en cuenta tanto aspectos internos como aquellos propios del ambiente. Por otra parte, los enfoques más integradores consideran que el estrés debe comprenderse desde una perspectiva más amplia, por cuanto en este convergen e interactúan muchas variables.

Navas (citado por Naranjo, 2004a) coincide con esta última posición, pues considera que el estrés no es algo que pertenece solo a la persona o al ambiente, ni es tampoco un estímulo o una respuesta, sino que más bien es una relación dinámica entre la persona y el ambiente. Esto significa que la persona no es una víctima pasiva del estrés, y que su forma de interpretar los acontecimientos y la manera de valorar sus propios recursos y posibilidades para enfrentarlos, determina en gran medida la magnitud de la experiencia de este. Dependiendo de la forma en que la persona piense y de los sentimientos e imágenes que tenga respecto de una situación, puede crear, aumentar, mantener o disminuir la respuesta a él. Se puede decir entonces que, de acuerdo con la literatura científica, el estrés implica cualquier factor externo o interno que induce a un aumento en el esfuerzo por parte de la persona para mantener un estado de equilibrio dentro de sí misma y en relación con su ambiente.

El estrés se caracteriza por una respuesta subjetiva hacia lo que está ocurriendo; indica Arellano (2002), en este sentido que es el estado mental interno de tensión o excitación. Por otra parte, Melgosa (1995) señala que, debido a las alteraciones que 
el estrés provoca en la persona, se puede entender este concepto como: "un conjunto de reacciones fisiológicas y psicológicas que experimenta el organismo cuando se lo somete a fuertes demandas" (p. 19).

En cuanto a la tradición psicosocial del estudio del estés, en esta se da énfasis a las reacciones psicológicas y conductuales de las personas ante las situaciones estresantes que éstas enfrentan en su ambiente. Según esta teoría psicosocial, las personas se esfuerzan continuamente y de diferente manera para afrontar cognitiva y conductualmente las demandas tanto internas como externas de la situación valorada como estresante.

De acuerdo con Folkman, Lazarus, Gruen y De Longis (1986), diversos eventos de la vida tienen la propiedad de ser factores causantes de estrés, los cuales provocan un desequilibrio emocional. El estrés se presenta cuando la persona identifica una situación o un encuentro como amenazante, cuya magnitud excede sus propios recursos de afrontamiento, lo cual pone en peligro su bienestar. Se presentan de esta manera dos procesos: una valoración cognitiva del acontecimiento y un proceso de afrontamiento. En el primero, la persona valora si la situación puede dañarla o beneficiarla, es decir, cuánto y cómo repercute en su autoestima. En el segundo, estima lo que puede hacer o no para enfrentar la situación, para prevenir un daño o mejorar sus perspectivas.

Los autores mencionados, al realizar una investigación sobre la relación existente entre el proceso de afrontamiento y la valoración cognitiva, encontraron que los tipos de afrontamiento varían dependiendo de lo que las personas estimen que está en juego y de las opciones y recursos de cambio que poseen. La investigación mostró que cuando las personas consideraban que su propia autoestima estaba siendo amenazada, empleaban estrategias de confrontación, autocontrol, evitación y había un aumento de responsabilidad. En general, si estimaban que eran capaces de modificar la situación dando énfasis al aspecto cognitivo, usaban más estilos confrontativos, revaloraciones positivas y un plan de solución; por otra parte, si consideraban que eran incapaces de cambiar la situación, acentuaban las respuestas emocionales, empleando las estrategias de distanciamiento, escape o evitación.

\section{Eustrés, distrés y nivel óptimo de estrés}

Neidhardt, Weinstein y Conry (1989) mencionan que el doctor Hans Selye, considerado un experto en temas del estrés, definía este como la proporción de deterioro y agotamiento acumulado en el cuerpo. Un estrés excesivo debido a un estímulo demasiado grande, puede conducir a la angustia; es decir, al distrés. Se rompe la armonía entre el cuerpo y la mente, lo que impide responder de forma adecuada a situaciones cotidianas. Por otra parte, se utiliza el término eustrés, para definir la situación en la que la buena salud física y el bienestar mental facilitan que el cuerpo en su conjunto adquiera y desarrolle su máximo potencial. El estado de eustrés se asocia con claridad mental y condiciones físicas óptimas. Tomando en cuenta los aspectos mencionados, Neidhardt et al. (1989) definen el estrés como: "un elevado nivel crónico de agitación mental y tensión corporal, superior al que la capacidad de la persona puede aguantar y que le produce angustia, enfermedades, o una mayor capacidad para superar esas situaciones (eustrés)" (p. 17).

También, Bensabat (1987) se refiere a este tema y cita asimismo a Selye, indicando que para este autor, el estrés es también una respuesta no específica del organismo a toda demanda que se le haga. Esta definición, comenta, es muy amplia y significa que cualquier demanda, independientemente de que sea física, psicológica o emocional, positiva o negativa, provoca una respuesta biológica del organismo, idéntica y estereotipada. Esta respuesta es mensurable y corresponde a 
unas secreciones hormonales responsables de las reacciones al estrés, somáticas, funcionales y orgánicas.

Lo anterior implica que una emoción agradable producto de una buena noticia produce los mismos efectos que una emoción desagradable, producto de una mala noticia. Ambas situaciones son causa de estrés. No obstante, la mayoría de las veces, las respuestas del organismo se realizan en armonía, con la mayor naturalidad y sin consecuencias, ya que están adaptadas a las normas fisiológicas de la persona. Se trata del buen estrés o eustrés. Pero otras veces, las respuestas exigidas por una demanda intensa y prolongada, agradable o desagradable, son excesivas y superan las capacidades de resistencia y de adaptación del organismo. En este caso se trata de un mal estrés o distrés.

El buen estrés es todo aquello que causa placer, todo lo que la persona quiere o acepta hacer en armonía consigo misma, con su medio y con su propia capacidad de adaptación. Es el estrés de la realización agradable. Ejemplos de buen estrés son la alegría, el éxito, el afecto, el trabajo creador, un rato de tranquilidad, compartir con otras personas, o sea, todos los aspectos que resultan estimulantes, alentadores, fuentes de bienestar, de felicidad o de equilibrio.

El mal estrés es todo aquello que disgusta, todo cuanto la persona hace a pesar suyo, en contradicción consigo misma, su ambiente y su propia capacidad de adaptación. Son ejemplos de mal estrés la tristeza, el fracaso, las malas noticias, la enfermedad, las presiones, las frustraciones, la carencia de libertad, que son fuentes de desequilibrio, de alteraciones psicosomáticas y de enfermedades de adaptación.

El tipo de estrés depende también de cómo se reciben y se interpretan los acontecimientos. Una misma situación puede significar un mal estrés para una persona y un buen estrés para otra; o sea, lo que importa no es lo que sucede, sino la forma como se percibe. En cuanto al nivel óptimo de estrés, señala Bensabat (1987) que éste se refiere a la dosis de estrés biológicamente necesaria para cada persona, con el propósito de que ésta se desempeñe de manera armónica en unas condiciones óptimas compatibles con su personalidad y sus posibilidades de adaptación. El estrés es indispensable en la vida, es la consecuencia de las actividades de las personas. No obstante, si la dosis de estrés no es la óptima, ya sea por exceso o por carencia, el estrés se convierte en distrés y exige una adaptación particular del organismo por encima de las normas fisiológicas, tanto en el aspecto biológico como en el físico y en el psicológico.

Cuando la persona funciona por debajo del umbral óptimo del estrés, el organismo está subestimado, poco solicitado física, psicológica y biológicamente. Si el reposo es excesivo, se traduce en enojo y fatiga. Por el contrario, cuando se funciona por encima del nivel óptimo, el organismo se encuentra superestimulado, agotado por cansancio, demasiado solicitado, sometido a una adaptación excesiva que exige una secreción importante de hormonas de adaptación (adrenalina y cortisona), responsables de efectos secundarios y de alteraciones metabólicas y orgánicas.

A cada persona le conviene conocer tanto su nivel de estrés, sus límites y capacidad de adaptación así como la dosis de presión que puede soportar, para evitar situarse en condiciones que superen su umbral de tolerancia al estrés. Al respecto, Melgosa (1995) señala que una cantidad moderada de tensión vital, esto es, de estrés, resulta saludable, por cuanto ayuda a alcanzar metas elevadas y a resolver problemas difíciles. Menciona que:

\footnotetext{
Se ha comparado con acierto el estrés a las cuerdas de una guitarra, que necesitan la tensión justa para emitir su sonido con precisión. Las cuerdas afinadas de la guitarra sonarán maravillosamente en las manos de un músico experto, porque mantiene una cierta tensión. Esas mismas cuerdas, si están flojas, aún en las manos del mejor guitarrista, no sonarían, o su sonido resultaría repelente.... Sin embargo, si las tensamos demasiado, el sonido resultará igualmente desagradable. Además alguna cuerda acabará rompiéndose, e inutilizaría la guitarra (pp. 25-26).
} 


\section{Fases del estrés}

Generalmente, el estrés no sobreviene de manera repentina. El organismo del ser humano posee la capacidad para detectar las señales de que se está ante un evento productor de este. Desde que aparece hasta que alcanza su máximo efecto, se pasa por tres etapas: alarma, resistencia y agotamiento (Melgosa, 1995).

En cuanto a la fase de alarma, constituye el aviso claro de la presencia de un agente estresante. Las reacciones fisiológicas ante este agente son las primeras que aparecen para advertir a la persona que debe ponerse en estado de alerta una vez percibida la situación; por ejemplo, cuando hay exceso de trabajo o estudio, esta fase puede hacerle frente y resolver esto de la mejor forma posible, con lo cual la verdadera señal de estrés no llega a materializarse. Solamente cuando la barrera estresante supera a la persona y esta se da cuenta de que sus fuerzas no son suficientes, puede decirse que toma conciencia del estrés existente, lo cual la sitúa así en la fase de alarma.

Cuando el estrés prolonga su presencia más allá de la fase de alarma, la persona entra en la segunda fase, denominada de resistencia. Intenta continuar enfrentado la situación, pero se da cuenta de que su capacidad tiene un límite y, como consecuencia de esto, se frustra y sufre. Empieza a tomar conciencia de que está perdiendo mucha energía y su rendimiento es menor, lo cual la hace tratar de salir adelante, pero no encuentra la forma; esto hace que esta situación se convierta en un círculo vicioso, sobre todo cuando va acompañada de ansiedad por un posible fracaso.

Posteriormente, se presenta la fase de agotamiento que es la fase terminal del estrés. Esta se caracteriza por la fatiga, la ansiedad y la depresión, las cuales pueden aparecer por separado o simultáneamente. La fatiga incluye un cansancio que no se restaura con el sueño nocturno, y generalmente va acompañada de nerviosismo, irritabilidad, tensión e ira. Respecto de la ansiedad, la persona la vive frente a una diversidad de situaciones, no solo ante el agente estresante, sino también ante experiencias que normalmente no se la producirían. En cuanto a la depresión, la persona carece de motivación para encontrar placenteras sus actividades, sufre de insomnio, sus pensamientos son pesimistas y los sentimientos hacia sí misma son cada vez más negativos.

Es importante tomar conciencia de que todas las personas tienen un límite y que, si este es respetado, tendrán la oportunidad de que tanto su calidad como esperanza de vida se vean mejoradas.

\section{La respuesta al estrés}

Como se mencionó anteriormente, se han identificado tres fases en la respuesta al estrés: alarma, resistencia y agotamiento. La alarma, según explican Neidhardt et al. (1989), se produce cuando el cerebro percibe un factor causante de estrés. El cerebro envía de inmediato un mensaje a la glándula pituitaria que inicia la segregación de una hormona, la cual induce a que otras varias glándulas inicien la producción de adrenalina. El efecto consiste en poner a todo el organismo en un estado general de alerta. Las señales más aparentes de que se han activado los sistemas de respuesta son: pulso rápido, aumento de la sudoración, corazón que palpita fuertemente, estómago contraído, brazos y músculos de las piernas en tensión, respiración entrecortada y rápida, dientes apretados con firmeza, mandíbulas cerradas, incapacidad para permanecer quieto y emociones intensas.

Cuando la persona manifiesta esos síntomas, es indicativo de que está preparada para enfrentar un peligro, amenaza o situación comprometida, sea real o imaginaria. No obstante, este estado es pasajero y reservado solo para reaccionar ante situaciones extremas, puesto que el organismo no podría mantenerlo como un estado duradero. 
Melgosa (1995) explica la fisiología del estrés mencionando que todas las señales de alarma que llegan al cerebro son enviadas al hipotálamo, el cual es un pequeño órgano situado en el centro de la masa cerebral. El hipotálamo transmite estos mensajes a todo el organismo por vía nerviosa y por vía sanguínea.

Por vía nerviosa, los estímulos producidos por el hipotálamo se transmiten al sistema nervioso simpático, que regula las funciones orgánicas. Estos estímulos llegan a producir alteraciones en el funcionamiento de los órganos y también alcanzan a la médula de las glándulas suprarrenales, lo que provoca un aumento en la secreción de adrenalina y noradrenalina, las cuales pasan a la sangre y producen también alteraciones sobre todo el organismo.

En cuanto a la vía sanguínea, el hipotálamo estimula a la hipófisis, la cual segrega diversas hormonas que, al pasar a la sangre, actúan sobre todo el organismo. La hormona más importante de las que segrega la hipófisis cuando es estimulada por el hipotálamo es la corticotropina (ACTH u hormona del estrés), la cual hace que en la corteza de las glándulas suprarrenales se produzca otra hormona, la cortisona, que produce numerosos efectos y alteraciones sobre el organismo. Otras hormonas segregadas por la hipófisis actúan sobre la glándula tiroides, los testículos o los ovarios provocando efectos importantes sobre muy diversos órganos.

Además de las respuestas físicas, existen otras respuestas ante el estrés. Neidhardt et al. (1989) las categorizan en dos grupos: las psíquicas y las de conducta. Respecto de las respuestas psíquicas, las más generalizadas son: incapacidad para concentrarse; dificultad para tomar decisiones, incluso las más sencillas; pérdida de la confianza en sí mismo o en sí misma; irritabilidad; preocupaciones y ansiedad; temores irracionales o pánico sobrecogedor.

El estrés con frecuencia viene acompañado de emociones como exaltación, depresión o ira, de una intensidad variable dependiendo de la situación. Cuando esto ocurre, lo más apropiado es expresar esas emociones, lo que ayuda a superar el estrés. Las emociones no expresadas o reprimidas, sean positivas o negativas, generalmente producen estrés.

En cuanto a las respuestas de conducta, se mencionan algunas como: aumento en el empleo de medicamentos, tics nerviosos, distracción, proclividad a los accidentes, comer en exceso o falta de apetito, dormir demasiado o insomnio, aumento en el consumo de alcohol o drogas, impulsividad y agresividad.

También Melgosa (1995) se refiere a este tema de las respuestas al estrés, las cuales sitúa en tres grandes categorías: cognitivas, emotivas y conductuales. A continuación se explican.

\section{Área cognitiva (pensamientos e ideas)}

La persona tiene dificultad para permanecer concentrada en una actividad y presenta una frecuente pérdida de atención. La retención memorística se reduce, tanto en la memoria a corto plazo como a largo plazo. Los problemas que exigen una reacción inmediata y espontánea se resuelven de una manera impredecible. Cualquier problema que requiera actividad mental tiende a solucionarse con un número elevado de errores. Por lo general, la persona se siente incapaz de evaluar acertadamente una situación presente y tampoco puede acertar a proyectarla en el futuro. Además, la manera de pensar no sigue patrones lógicos y coherentes dentro de un orden, sino que se presenta desorganizada.

\section{Área emotiva (sentimientos y emociones)}

La persona experimenta dificultad para mantenerse relajada tanto física como emotivamente. Aparte de los desajustes físicos reales, se empieza a sospechar de nuevas enfermedades (hipocondría), aparecen rasgos como el desarrollo de la impaciencia, la intolerancia, el autoritarismo y la falta 
de consideración por otras personas. Los principios morales que rigen la vida de la persona se relajan y se posee menor dominio propio. Hay un aumento de desánimo y un descenso del deseo de vivir. La autoestima también se ve afectada por pensamientos de incapacidad y de inferioridad.

\section{Área conductual (actitudes y comportmientos)}

En el lenguaje se presenta una incapacidad para dirigirse oralmente a un grupo de personas de forma satisfactoria, puede darse tartamudez y un descenso de fluidez verbal. La persona experimenta falta de entusiasmo por las aficiones preferidas, así como por sus pasatiempos favoritos. Es frecuente el ausentismo laboral y escolar, así como un aumento del consumo de alcohol, tabaco, café u otras drogas. El nivel de energía disponible fluctúa de un día para otro. Los patrones de sueño se alteran. Generalmente se sufre de insomnio y se llega a veces a una extremada necesidad de dormir. En cuanto a las relaciones interpersonales, aumenta la tendencia a la sospecha, se tiende a culpar a las otras personas o a atribuirles responsabilidades propias. También hay cambios en la conducta, tales como reacciones extrañas y la aparición de tics, o sea, actitudes que no sean propias de la persona. Incluso pueden manifestarse ideas suicidas e intentos de llevarlas a cabo.

Se considera que cuando una persona se encuentra bajo el efecto del estrés puede aumentar su capacidad de percepción, de memoria, de razonamiento y de juicio durante un periodo de tiempo limitado; no obstante, cuando la tensión se lleva más allá del límite, sobreviene el declive y se observan dificultades en las capacidades cognitivas, tales como la dificultad para concentrarse y la pérdida de memoria. Esta situación puede apreciarse en estudiantes que acuden a un examen y, debido a la tensión del momento, no logran responder a las preguntas. Asimismo, la capacidad de razonar, de resolver problemas o de emitir juicios se ve menoscabada por la presencia del estrés. Ocurre cuando la persona dice encontrarse "bloqueada" y dice no poder ni saber cómo reaccionar ante una situación.

Otra reacción común de la persona sometida a estrés es la frustración. Este estado anímico la irrita; de hecho, diversos especialistas vinculan la frustración con la agresividad. Asimismo, con mucha frecuencia la persona estresada presenta síntomas de ansiedad, tales como la aprehensión, la preocupación, la tensión y el temor por el futuro. Sobre la ansiedad, señala Melgosa (1995):

La ansiedad es una de las más peligrosas manifestaciones psicológicas del estrés. Es normal experimentar cierto grado de ansiedad ante las situaciones inciertas; pero la ansiedad excesiva es una forma de neurosis que no beneficia ni al sujeto ni a los que con él conviven. Además, no hemos de olvidar la estrecha vinculación que existe entre estrés y ansiedad, y que muchas personas, que en la actualidad sufren los efectos devastadores de la ansiedad, iniciaron sus "hábitos" ansiosos en medio de situaciones estresantes (p. 40).

En cuanto a la depresión, se considera que es uno de los riesgos terminales del estrés. Cuando este ha continuado más allá de la fase de alarma y se ha mantenido constante durante un tiempo prolongado en la fase de resistencia, se entra en la fase de agotamiento, en la cual el resultado más próximo es la depresión. La persona deprimida ya no realiza proyectos ni siente deseos de proponerse ninguno y el inicio de toda jornada le resulta demasiado pesado (Bensabat, 1987). En síntesis, la dolorosa pérdida de la autoestima, sin perspectiva alguna de modificación, es un estado que se torna permanente.

Otra forma de reacción al estrés son las enfermedades psicosomáticas. $\mathrm{Al}$ respecto, las terapias de la conducta consideran que ciertas personas son capaces de provocar en ellas mismas reacciones nocivas de estrés de acuerdo con su forma de pensar, de percibir y de responder al mundo exterior y a la relación con otras personas. 
Un enfrentamiento inadecuado del estrés llega hasta la aparición de síntomas y trastornos psicosomáticos (Bensabat, 1987). Sobre este tema Melgosa (1995) explica que: "se entiende por trastornos, reacciones o enfermedades psicosomáticas, aquellas dolencias que dan manifestaciones orgánicas, pero tienen su origen en causas psicológicas, como el propio estrés u otros estados mentales adversos" (p. 44).

Además, este autor señala que prácticamente todos los órganos vitales del cuerpo humano pueden, directa o indirectamente, verse afectados por los estados emocionales. Debido a esto, es amplia la gama de dolencias psicosomáticas; por ejemplo: cutáneas, alopecia (caída del pelo), acné, urticaria, psoriasis. Del aparato locomotor: dolores de espalda, calambres musculares, reumatismo. Respiratorias: asma, alergia, rinitis, bronquitis. Genitourinarias: vaginismo, impotencia, síndrome premenstrual, micciones dolorosas. Endocrinas: hipertiroidismo, obesidad. Nerviosas: ansiedad, debilidad, dolores musculares, cefaleas, tics. De los ojos: conjuntivitis.

\section{Causas del estrés}

Cualquier situación que la persona perciba como una demanda o una amenaza o que exija un rápido cambio es productora de estrés. No obstante, debe recordarse que no todas las situaciones ni todos los factores estresantes son negativos. Por ejemplo, obtener un buen resultado en una prueba académica o ser ascendido en el trabajo pueden causar estrés de una forma positiva. Además, dependiendo de la forma en que se perciban los diversos factores estresantes, se producen distintos impactos en las personas.

En general, puede decirse que existen dos grandes fuentes fundamentales del estrés. En primer lugar, están los agentes estresantes que provienen de circunstancias externas como del ambiente, de la familia, del trabajo, del estudio, entre otras.
En segundo lugar, se encuentra el estrés producido por la persona misma, es decir, por sus propias características de personalidad y por su forma particular de enfrentar y solucionar los problemas.

Por otra parte, los factores de estrés no siempre son conocidos, y puede que sean desconocidos, subestimados, inconscientes y descubiertos solamente después de un diálogo o un análisis (Bensabat, 1987). Las angustias, las fobias y las obsesiones son causas de estrés. Su origen es con frecuencia inconsciente y va unido a antiguos traumatismos físicos o psico-afectivos. Los trastornos mentales, las depresiones y la neurosis son a la vez una fuente de estrés y el resultado de situaciones causantes de este, las cuales pueden ser conscientes o generalmente inconscientes y se remontan hasta la infancia. La frustración, estar pendiente constantemente de un asunto, el temor, el recelo, el sentimiento de insatisfacción, la ausencia de motivación y de sentido de la vida son otras causas con frecuencia de origen inconsciente y responsables de una tensión psicológica constante.

Diferentes autores asocian los distintos tipos de afrontamiento al estrés de acuerdo con las diferencias en los rasgos de personalidad. Holahan y Moss (1986) mencionan que la autoestima, el autodesprecio, el dominio y la buena disposición son ejemplos de rasgos de la personalidad que desempeñan un papel importante frente a las situaciones estresantes.

Friedman y Rosenman (citados por Melgosa 1995 y Bensabat, 1987) establecieron la distinción entre dos tipos de personalidades, a las que denominaron el tipo A y el tipo B; asimismo, posteriormente se menciona un tipo C. Por supuesto existe una gama de tipos intermedios con características de los distintos tipos, pero esta simplificación resulta útil para que la persona pueda determinar hacia qué tipo de personalidad se inclina más.

El tipo A es extrovertido; corresponde a las personas que exteriorizan su respuesta al estrés mediante reacciones 
excesivas. La menor contrariedad se traduce en irritabilidad y en excesos importantes y desproporcionados de cólera en relación con los factores estresantes. Este tipo de personalidad corresponde a personas ambiciosas e impulsivas que viven constantemente bajo tensión, asumen una actitud de competencia con el medio y con ellas mismas queriendo siempre hacer más y ser mejores; son perfeccionistas, siempre se conducen con prisa, pendientes del reloj, preocupadas por el tiempo y los plazos de vencimiento. Estas personas poseen un sentido agudo de la realidad, son impacientes crónicos, se empujan a sí mismas y a las demás, tienen dificultades para relajarse, se enfrascan en sus trabajos, sacrificando muchas veces otras dimensiones de su vida, como la familiar y la afectiva.

La persona con el tipo de personalidad C, por el contrario, es introvertida y obsesiva. Corresponde a la persona que interioriza su reacción al estrés. Aparentemente son resignadas y apacibles, pero se trata de falsas calmas, puesto que sufren en silencio.

El tipo de personalidad B es intermedio, el modo ideal de comportamiento. Corresponde a la persona que domina de forma adecuada y natural su estrés, reacciona con calma, con prudencia y con una buena perspectiva de la situación. Su carácter es optimista. Este tipo de personalidad es minoritario, mientras que los tipos A y C son más frecuentes entre la población.

Las personas del tipo A están más predispuestas a la patología cardiovascular, hipertensión arterial, angina de pecho, infarto del miocardio y hemorragia cerebral. También tienen más predisposición a padecer de úlceras gastroduodenales y obesidad, la cual se debe, con mucha frecuencia, a una compensación alimentaria de las frustraciones y el nerviosismo. Las personas del tipo C están más predispuestas a la depresión nerviosa, a los reumas, a las infecciones, a la alergia y presentan muchas veces una disminución importante de las defensas inmunitarias. Las personas del tipo B son naturalmente las más protegidas.
Algunas conductas típicas de la personalidad de tipo A son el movimiento constante, la impaciencia, una expresión facial tensa, generalmente se siente insatisfecha con su situación, es competitiva, se queja con frecuencia, su conversación es rápida y a un volumen alto, con altibajos y énfasis, expresiva y gesticulante, responde de inmediato, la respuesta es breve y directa, apresura al interlocutor o lo interrumpe con frecuencia. Por el contrario, en la personalidad de tipo B la conducta es de tranquilidad motriz, de calma, la expresión facial es relajada, la risa suave, la persona está generalmente satisfecha con su situación, evita las situaciones competitivas y rara vez se queja, su conversación es pausada y a bajo volumen, en tono uniforme, con gesticulación moderada, responde tras una pausa, ofrece respuestas más bien extensas, escucha con atención y espera para responder.

Navas (citado por Naranjo, 2004a) menciona algunas recomendaciones que se sugieren para modificar conductas de la personalidad de tipo A, entre ellas: aprender a organizar el tiempo, especialmente el de la "enfermedad de la prisa", no anteponer el ego ante lo que se hace para evitar sentimientos hostiles, planificar el trabajo, desarrollar pasatiempos, el hábito de la lectura, la recreación y el contacto con la naturaleza, participar en actividades donde no se tenga que estar compitiendo, aprender a delegar tareas y dar tiempo razonable a las demás personas para que las realicen, comer disfrutando de la comida y aprender a relajarse.

En cuanto a las causas del estrés, Melgosa (1995) hace referencia a experiencias traumáticas, las molestias cotidianas, el ambiente físico y social, las elecciones conflictivas y algunas circunstancias estresantes típicas que se presentan a lo largo de la vida.

Las experiencias traumáticas, tanto si provienen de catástrofes naturales como terremotos o huracanes, así como si son provocadas por humanos, tales como accidentes o agresiones, aportan una 
cantidad considerable de estrés durante y después del acontecimiento. Las personas víctimas de experiencias traumáticas pasan por un periodo de estrés que sigue tres pasos: el primero es una ausencia de respuesta, la persona parece ida, como ajena a lo ocurrido; el segundo paso se evidencia por la incapacidad para iniciar una tarea por simple que sea; en el tercer paso, finalmente, la persona entra en una etapa de ansiedad y aprehensión en la que revive las escenas mediante fantasías o pesadillas. Algunas veces puede sufrir un agudo sentimiento de culpabilidad, sobre todo en aquellos casos en que otras personas han perdido la vida.

Asimismo, las molestias cotidianas tales como perder el autobús o llegar tarde a una cita crean tensión. Si estas situaciones se presentan con mucha frecuencia y la persona permite que la afecten, se puede llegar a niveles de estrés muy altos, como si este fuera producto de un evento estresante de gran magnitud. También el ambiente físico que rodea a las personas contribuye a generar, en mayor o menor grado estrés; por ejemplo, el exceso de ruido y la escasez de espacio vital.

Otro factor causante de estrés es cuando la persona tiene que decidir entre dos o más opciones. Independientemente de si estas son negativas o positivas, es su similitud la que crea la dificultad de elección. Dependiendo de la cualidad positiva o negativa de las opciones, se han identificado cuatro tipos de conflictos que surgen a la hora de elegir, descritos a continuación.

El primero es el conflicto de evitación-evitación. Se trata de la presencia de dos situaciones que se perciben como negativas y que sumergen a la persona en un conflicto por el cual trata de evitar ambas; no obstante, en la mayoría de los casos tiene que optar por una, probablemente la menos perjudicial. El segundo conflicto es el de atracción-atracción. En ésta ambas situaciones se perciben como positivas y se desean a la vez, pero igualmente la persona debe decidirse por una de ellas. El tercer conflicto es el de atracción-evitación. Este tipo de conflicto ocurre ante la presencia de una situación que se percibe al mismo tiempo como positiva y negativa. Por ejemplo, obtener una beca para estudiar en el extranjero que implica dejar a la familia. El cuarto y último conflicto es el de doble atracción-evitación. Este aparece como resultado de la existencia de dos situaciones, cada una de ellas con componentes positivos y negativos a la vez.

Para tomar decisiones se recomienda no actuar o decidir impulsivamente, anotar las opciones posibles, analizar las ventajas e inconvenientes de cada una de ellas, proyectar las opciones en el futuro inmediato, seleccionar la opción que se considere como la más provechosa y aceptarla con sus consecuencias.

Diversos autores consideran también las relaciones humanas como una causa central del estrés, debido a la necesidad del ser humano de afecto y pertenencia. Kaplan (citado por Hays y Oxley, 1996) menciona que aquellas personas que logran mantener relaciones adecuadas con otras tienen menor tendencia de padecer enfermedades físicas y psicológicas. Cuando ocurre la situación contraria, las personas presentan problemas de adaptación al ambiente y al afrontar el estrés. El fracaso en los intentos de comunicación lo genera cuando se escapan datos y detalles importantes, cuando se expresa algo pero no se consigue que la otra persona comprenda exactamente lo que desea, cuando se dice sí y lo que se quería decir era no o viceversa; es decir, no se logra una comunicación asertiva y clara. Los ejemplos anteriores son de situaciones que provocan que la persona se sienta confundida y actúe con angustia y tensión.

\section{Prevención del estrés}

Para poder prevenir y afrontar el estrés de manera óptima, el primer requisito es que la persona se fortalezca física y psicológicamente, de manera que 
pueda hacer frente y resistir las inevitables circunstancias de la vida. Es importante también que aprenda a disminuir el nivel de estrés de las situaciones personales, de modo que no superen las propias resistencias. Otro factor relevante para lograr el éxito ante al estrés es la actitud o la forma en que la persona asuma y analice las situaciones que enfrenta.

Sobre este tema de las actitudes ante situaciones que provocan estrés, Lazarus y Folkman (1986) plantean que las personas evalúan los agentes estresantes de tres formas distintas. La primera opción es considerar al agente como un daño irreparable ya ocurrido. En este caso, la persona sufre el riesgo de permanecer lamentándose del pasado y obstruir toda estrategia reparadora. Por otra parte, si percibe la situación como una amenaza tiende a ver el futuro con pesimismo, de forma incierta y es probable que la ansiedad se apodere de ella. Finalmente, si la persona asume el agente estresante como un reto, se sentirá con mayor capacidad para enfrentar la situación y buscar posibles alternativas de solución.

Otros aspectos a los que se debe prestar atención para prevenir el estrés, de acuerdo con autores tales como Bensabat (1987), Melgosa (1995) y Neidhardt et al. (1989), son, por ejemplo: el ejercicio físico y el reposo, la alimentación, el contacto con la naturaleza, el tiempo libre, la planificación del tiempo, objetivos y actividades.

En cuanto al ejercicio y el reposo, se menciona que la mayoría de las personas que sufren de estrés no practican ejercicio físico ni son capaces de lograr un descanso adecuado, lo cual genera un círculo vicioso de difícil salida. Empezar a practicar ejercicio es la forma más sencilla y eficaz de salir de ese círculo y, además, el reposo necesario para recuperar la energía se facilita cuando se realiza ejercicio de manera regular.

El ejercicio físico proporciona una serie de beneficios opuestos a la acción del estrés, entre ellos: aumenta la eficacia del corazón; mejora la circulación sanguínea; reduce el nivel de colesterol; favorece la producción de endorfinas, que son responsables del estado de bienestar general; relaja los músculos; ayuda a mantener la flexibilidad de las articulaciones; quema el exceso de energía permitiendo mantener el peso ideal; favorece la oxigenación de las células y ayuda a controlar la hipertensión; asimismo, facilita el descanso, favorece la eliminación de toxinas, aumenta la capacidad y agilidad mental, mejora el estado de ánimo y favorece el buen humor y la autoestima.

Sobre el descanso, este debe ser cotidiano, semanal y anual. Además, debe ser de calidad, de modo que permita recuperarse del desgaste tanto físico como psíquico que conlleva la realización de las actividades.

Los buenos hábitos relacionados con la alimentación son importantes siempre, pero sobre todo cuando hay estrés, esto por cuanto si la persona está sufriendo de este tiende a comer en exceso, a no comer lo necesario o hacerlo deprisa y en cualquier momento. Además, cuando el organismo se encuentra estresado, emplea una mayor cantidad de energía y de manera rápida, lo cual añade una carga al sistema cardiovascular.

Algunas recomendaciones sobre hábitos alimenticios son: comer despacio masticando correctamente los alimentos y a horas fijas; controlar la cantidad de grasa que se ingiere; consumir frutas, verduras y cereales integrales en cantidad suficiente; reducir la ingesta de azúcar, sal y condimentos; asegurarse de ingerir vitaminas del complejo B por cuanto son fundamentales para conservar el equilibrio nervioso y una buena función cerebral, al igual que de la vitamina $\mathrm{C}$; reducir al mínimo el consumo de productos que contengan grasa animal para reducir el nivel de colesterol; controlar el peso y tomar suficiente agua, de la cual se sugiere tomar entre un litro y litro y medio fuera de las comidas y aparte de la que por su propia naturaleza contienen los alimentos.

El contacto con la naturaleza es una experiencia relajante integral que ayuda a 
prevenir el estrés. La persona obtiene de la interacción con ella paz y salud. La naturaleza ofrece múltiples beneficios, entre ellos la vista se recrea con el paisaje y los colores, el oído con los sonidos naturales, el olfato con los olores silvestres, el gusto con alimentos saludables tomados de este ambiente y el organismo entero con el aire puro y el sol.

Utilizar de forma adecuada el tiempo libre permite también prevenir de mejor forma el estrés, por cuanto compensa los efectos negativos del exceso de trabajo o de estudio, ayuda a afianzar las relaciones familiares y sociales y permite cambiar el ritmo y dejar de lado los problemas cotidianos.

La planificación personal también ayuda a prevenir el estrés. Se sugieren dos pasos: primero, identificar los objetivos personales clasificando lo que para la persona es realmente importante, mediante el establecimiento de una escala de prioridades. Y segundo, organizar el tiempo disponible de la mejor manera elaborando, por ejemplo, un programa realista de actividades que permita cumplir con estas sin un exceso de presión.

Bensabat (1987) ofrece algunas sugerencias prácticas anti-estrés, entre ellas: hacer un inventario de los factores habituales de estrés y tratar de eliminar los que son responsables de tensiones inútiles; sustraerse de allegados o familiares cuando se sienta la necesidad de ello; no dudar en interrumpir una reunión o entrevista cuando se sienta demasiada presión; realizar pausas durante la jornada de trabajo o de estudio, o sea, aislarse, relajarse y no pensar en nada; no tratar de complacer a todas las personas, pues de todos modos no se logrará; no participar en situaciones que desagraden; pensar más en sí mismo o en sí misma y procurarse bienestar; desarrollar un pensamiento positivo y constructivo de las cosas; no guardarse sentimientos y pensamientos que se desean expresar; no conceder a las cosas más importancia de la que tienen, puesto que es una pérdida de tiempo y de energía; aprender a aceptar que algunas veces se pierde; amar lo que se hace y vivir al ritmo de la propia capacidad de adaptación.

\section{Afrontar el estrés}

El estrés puede ser controlado. La persona puede adiestrarse para desarrollar determinadas habilidades que le ayuden a identificar factores potenciales de estrés y a modificar reacciones perjudiciales ante él.

Una de las habilidades que se pueden desarrollar es la de lograr relajarse. La relajación proporciona resultados muy satisfactorios en el tratamiento del estrés. Además del beneficio inmediato que brinda, otorga a la persona la sensación de estar en control de sí misma, de que el estado de tensión no se escapa de su voluntad y que por lo tanto es una situación controlable. La práctica de la relajación concede una sensación placentera de gran tranquilidad mental y distensión muscular completa. Cuando la tensión es muy fuerte, se requiere que la relajación sea profunda para que resulte eficaz. No obstante, pese a sus múltiples beneficios, tiene el inconveniente de que no puede practicarse de manera inmediata cuando se presenta una situación productora de estrés. En estos casos, se recomienda una forma de relajación rápida basada en la respiración, la cual consiste en tres pasos: de primero se debe respirar profundamente reteniendo el aire tres o cuatro segundos y exhalándolo lentamente, lo cual se repite dos o tres veces; de segundo, la persona se repite mentalmente varias veces la frase: estoy tranquila (o tranquilo), me siento en calma; de tercero, se vuelve a respirar según la forma indicada.

Otra forma de controlar el estrés es aprender a preocuparse constructivamente. De acuerdo con Melgosa (1995), existen dos tipos de preocupación: la destructiva y la constructiva. La preocupación destructiva es aquella que no está provista de estrategias de solución, es repetitiva, recurrente y obsesiva. Puede centrarse en torno a un suceso del pasado que no puede cambiarse o a uno del 
futuro que es incierto. Es preocuparse por preocuparse y contribuye al empeoramiento de la situación. En cuanto a la preocupación constructiva, es racional, incluye planes y métodos para afrontar los agentes productores del estrés y, fundamentalmente, se centra más en las posibles soluciones que en el problema en sí.

Las cogniciones desempeñan un papel central en el afrontamiento del estrés. El modelo cognitivo plantea la hipótesis de que las percepciones que se tengan de los eventos influyen sobre las emociones y los comportamientos de las personas. De acuerdo con Beck y Ellis (citados por Naranjo, 2004a) las situaciones no determinan los sentimientos, sino el modo en que la persona las interpreta; es decir, la respuesta emocional está condicionada por la percepción de la circunstancia.

Autores como Beck, Emery y Greenberg (citados por Garrido y García, 1994) afirman que el organismo es un todo unitario formado por un grupo de subsistemas relacionados entre sí: afectivo, conductual, fisiológico y cognitivo. El sistema cognitivo es el que recibe y envía información a los otros subsistemas y regula el comportamiento de estos poniendo en marcha o inhibiendo ciertas respuestas de ellos en función del significado que le da a la información de que dispone. La evaluación negativa realizada por el sistema cognitivo provoca en los sistemas conductual y afectivo conductas y sentimientos negativos, de acuerdo con la interpretación hecha. Se presentan entonces conductas como la huida, la evitación o el dejar de realizar actividades; asimismo, emociones como apatía, tristeza, ansiedad y temor.

Beck (citado por Feixas y Miró, 1993) ha identificado una serie de lo que ha denominado errores cognitivos, los cuales favorecen la presencia no solo del estrés, sino también de depresión y ansiedad. Estos errores cognitivos son:

Pensamiento absolutista de todo o nada. Se manifiesta en la tendencia de ver las experiencias según dos posibilidades opuestas tomando una de ellas.
Sobregeneralización. Proceso de establecer una regla o conclusión general a partir de detalles que no la justifican por tratarse de hechos aislados que no se pueden aplicar a otras situaciones.

Filtro mental. Se trata del proceso de filtrar la experiencia de modo que se atiende solo a un detalle de la situación sin darse cuenta de otros aspectos, tal vez positivos, que suceden alrededor.

Descalificación de lo positivo. Se rechazan las experiencias positivas, insistiendo en que no cuentan por un motivo u otro. De esta forma se mantienen las creencias negativas.

Sacar conclusiones precipitadas. Esto ocurre sin disponer de datos suficientes que las apoyen.

Engrandecer o minimizar. Se exagera la importancia de unos acontecimientos en detrimento de otros.

Deberes e imperativos. Se trata de autoimposiciones que la persona se hace. Generalmente no son realistas y esta se exige más de lo que puede dar.

Personalización. Tendencia que tiene la persona de atribuirse a sí misma la responsabilidad de errores o hechos externos aunque no haya base para ello.

De acuerdo con Ellis y Abrahams (1980), una actividad cognoscitiva disfuncional acarrea reacciones emocionales y conductas de inadaptabilidad. Por otra parte, los pensamientos racionales, los sentimientos apropiados y la conducta afectiva favorecen la sobrevivencia y el bienestar de la persona. Ellis (citado por Garrido y García 1994) define irracional como: "cualquier pensamiento, emoción o comportamiento que conduce a consecuencias contraproducentes y autodestructivas que interfieren de forma importante en la supervivencia y felicidad del organismo" (p. 328).

De acuerdo con Ellis (citado por Naranjo, 2004a), existen una serie de creencias o ideas irracionales fundamentales, a saber: la persona necesita ser querida y aceptada por todas las demás; para que la 
persona sea aceptada, debe ser totalmente competente y alcanzar todas las metas que se proponga; cuando las cosas no resultan como se quiere, la situación se vuelve horrible y catastrófica; si algo parece peligroso hay que preocuparse por ello permanentemente; la felicidad humana se debe a factores sobre los que no se tiene control alguno; es más fácil evitar que afrontar ciertas dificultades y responsabilidades; la persona necesita depender de otras y requiere siempre de alguien más fuerte en quien confiar; existe una solución única y adecuada para cada problema y si no se encuentra se avecina una catástrofe.

Semejante al modelo cognitivo, la terapia racional-emotivo-conductual propuesta por Albert Ellis considera que, esencialmente, la persona misma produce sus emociones y conductas mediante su sistema de creencias y la forma en que interpreta o piensa acerca de los acontecimientos. De esta manera, las experiencias o circunstancias (A), no producen directamente los sentimientos y acciones (C), sino que estos son producidos, más bien, por el sistema de creencias, pensamientos o interpretaciones de las situaciones (B). Esto es lo que se conoce como el modelo ABC de las emociones y conductas de la persona en esta terapia.

Navas (citado por Naranjo 2004a) describe una serie de métodos mediante los cuales la persona puede lograr un mejor control del estrés, que son:

- Establecer las situaciones causantes del estrés. Aquellas que provocan suficiente malestar como para determinar que están afectando a la persona. Se sugiere no incluir más de cinco.

- Medir el nivel de estrés. Se puede emplear una escala de medición para cada una de las situaciones consideradas estresantes; por ejemplo: 0-2 poca dificultad, 3-5 moderada dificultad, 6-10 mucha dificultad.

- Cambiar el pensamiento. Para ello, se puede emplear el modelo descrito anteriormente del ABC. La persona se pregunta qué está pensando y qué interpretación está dando en el punto $\mathrm{B}$ a alguna situación en A que está causando o aumentando el nivel de estrés. El propósito principal es controlar lo que ocurre en la mente, lo cual permite poder enfrentar y tratar de modificar la situación de una forma más tranquila y racional.

- Disputa de pensamientos irracionales. Cuando la persona toma conciencia de que está pensando o interpretando de forma irracional una situación, debe disputarla creando frases, pensamientos o interpretaciones opuestas en términos más lógicos y apegados a la realidad.

- Relajación física y mental. Usando la imaginación y respiración adecuada mediante el empleo de las técnicas de relajación, la persona puede reducir el nivel de tensión en distintos momentos del día.

- Fomentar la autoestima. La persona tiene necesidad de sentirse valiosa. Cuando la autoestima es positiva, ayuda a enfrentar de una manera más adecuada aquellos eventos productores de estrés. Algunas sugerencias para mejorar la autoestima son: aprender a considerarse un ser valioso por la sola razón de existir; aprender a apreciar las fortalezas propias y no solo vivir repasando las debilidades; desarrollar relaciones con gente que permita a la persona sentirse apreciada y que la valore como un ser humano valioso.

- Aceptación incondicional. Esto implica que es necesario aprender a apreciar el valor personal como algo diferente a los actos y, al darse cuenta de que estos son erróneos, se intente modificarlos sin degradarse como persona.

- Comunicación asertiva. Para superar el estrés es necesario a aprender a emplear una comunicación clara. El desarrollo de la habilidad de 
comunicarse asertivamente facilita la relación con las otras personas, por tratarse de un estilo más adecuado, eficaz y saludable de comunicación.

- Tratar de modificar el tipo A de personalidad. Esto debido a que, como se mencionó anteriormente, corresponde a un estilo de comportamiento que frecuentemente causa el estrés como una respuesta.

\section{Estrés en la educación}

Diversos estudios se han centrado en las fuentes de tensión de la población estudiantil en las instituciones educativas. Entre estas fuentes se puede citar la inseguridad, la violencia física y psicológica, así como el sentirse inferior o incapaz. Señala Rice (2000) que cualquier situación que perturbe la armonía e interfiera en las actividades de la clase puede ser fuente de estrés y, ciertamente, afecta el funcionamiento académico en el acontecer educativo. Martínez y Díaz (2007) definen el estrés escolar como:

El malestar que el estudiante presenta debido a factores físicos, emocionales ya sea de carácter interrelacional o intrarelacional, o ambientales que pueden ejercer una presión significativa en la competencia individual para afrontar el contexto escolar en rendimiento académico, habilidad metacognitiva para resolver problemas, pérdida de un ser querido, presentación de exámenes, relación con los compañeros y educadores, búsqueda de reconocimiento e identidad, habilidad para relacionar el componente teórico con la realidad específica abordada (p. 5).

Además, desde el ingreso a la institución, la población estudiantil puede sufrir de estrés durante el período lectivo, puesto que se requiere de un proceso de adaptación, la ejecución de asignaciones extra clase, el cansancio cognitivo, los problemas de indisciplina, entre otros; situaciones que a su vez pueden ser indicadores de ciertos malestares en el ámbito escolar y el deseo de concluir el año lectivo.
Forero, López y Pardo (citados por Martínez y Díaz, 2007) se refieren a una investigación realizada por Witkin sobre el estrés infantil que señala a la escuela como una institución estresante de importancia, debido a aspectos tales como la competitividad por el rendimiento académico, las rivalidades entre compañeras y compañeros, la participación en el salón de clase, la realización de pruebas, el temor al fracaso y a decepcionar a madres y padres, y lograr la aceptación entre un grupo de iguales. Todas estas situaciones tienen además un impacto negativo en la autoestima. En otra investigación realizada por Dumont, LeClerc y Deslandes (2003), estos autores encontraron que un alto porcentaje de estudiantes de la muestra evidenciaban síntomas de ansiedad antes de presentar una prueba.

La ansiedad, muchas veces producto del estrés como se ha mencionado, es un sentimiento vago y altamente desagradable de temor y aprehensión. Es normal que el estudiantado se preocupe cuando enfrenta desafíos en la institución educativa, como por ejemplo rendir bien en un examen. De hecho, los investigadores han encontrado que estudiantes exitosos presentan niveles moderados de ansiedad; no obstante, algunas y algunos presentan altos niveles de ansiedad y se preocupan constantemente, lo cual puede afectar en forma significativa su habilidad de logro (Santrock, 2002).

Los niveles altos de ansiedad pueden ser también el resultado de las expectativas irreales y la presión de las madres y de los padres respecto del logro educativo de sus hijas e hijos. Muchas personas estudiantes sienten mayor ansiedad conforme alcanzan grados educativos superiores, en los que enfrentan evaluaciones más frecuentes, comparaciones sociales e incluso experiencias de fracaso. Cuando las instituciones educativas crean circunstancias como estas, es más probable que contribuyan a aumentar la ansiedad de la población estudiantil.

La presión que siente el estudiantado por obtener un buen rendimiento escolar puede provocarle una exacerbación de la 
ansiedad (Morris, 1997). La evidencia indica que las personas que sienten que no están en control de los sucesos estresantes de su vida son más propensas a experimentar trastornos de ansiedad. Indica este autor que cada vez con mayor frecuencia se observan alteraciones emocionales en estudiantes y también cómo la conducta disruptiva y la agresividad surgen de manera sorprendente.

Refiriéndose a este último tema, Jadue (2001) señala que el fracaso es fuente de frustración en una institución educativa que estimula la competitividad en sus estudiantes. La frustración es uno de los factores que puede llevar a la conducta agresiva, ya que fomenta la hostilidad entre estudiantes, de modo que cuando compiten por tener un buen rendimiento académico la violencia dentro de la escuela aumenta. Otros factores tales como la carencia de afecto, las situaciones económicas difíciles, la información masiva de los medios de comunicación y problemas del mismo ámbito educativo generan estrés.

Las relaciones conflictivas entre estudiantes y personal docente funcionan como otra situación estresante para la población estudiantil, la cual puede interferir en su desempeño académico exitoso. Esas relaciones conflictivas son conceptualizadas como interacciones discordantes y la falta de entendimiento entre estas partes (Universidad de Sevilla, 2005). Esta situación puede derivar en estudiantes que se desmotivan, además de desarrollar actitudes negativas hacia la institución educativa.

Newcomer (1993) menciona que los altos niveles de estrés y ansiedad reducen la eficiencia en el aprendizaje, ya que disminuyen la atención, la concentración y la retención, con el consecuente deterioro en el rendimiento educativo. A medida que va procesando la información, el estudiantado ansioso no logra organizar ni elaborar adecuadamente los contenidos de las materias y tiende a ser poco flexible para adaptarse a los procesos de aprendizaje. Es importante tomar en cuenta que, por su misma naturaleza, el estrés y la ansiedad dependen en gran medida del concepto que las personas tengan de las demandas que se les formulan en relación con la capacidad de comprensión y control que encuentran en sí mismas. Martínez y Díaz (2007) señalan que: "el estrés escolar se dimensiona en el desequilibrio psíquico, afectivo, cognitivo y social del estudiante" (p. 12).

Wigfield y Eccles (citados por Santrock, 2002) mencionan que se han creado diversos programas de intervención para reducir el alto nivel de estrés y ansiedad en estudiantes. Algunos han incorporado técnicas de relajación y otras estrategias que pretenden modificar los pensamientos negativos y autodestructivos y reemplazarlos por pensamientos positivos y enfocados en la tarea. Estos programas han mostrado ser efectivos tanto para reducir el estrés y la ansiedad como para mejorar el rendimiento académico de la población estudiantil.

\section{Conclusiones}

El estrés, sobre todo el crónico o distrés, tiene distintas repercusiones en la vida de la persona y afecta muchas veces su salud física y emocional, lo cual repercute a su vez en otras áreas de su existencia, tales como las relaciones interpersonales, el rendimiento laboral y académico y la vida familiar.

Las diferentes concepciones teóricas sobre el estrés enfatizan distintos aspectos de este. Algunas subrayan las respuestas orgánicas y otras se centran en factores externos generadores de reacciones psicológicas y conductuales ante él. Por otra parte, también hay concepciones que enfatizan en las valoraciones cognitivas que realizan las personas respecto de un evento estimando factores tanto internos como externos; además, están las perspectivas más integrales que consideran al estrés como una relación dinámica entre la persona y el ambiente.

Se establecen diferencias entre distrés y eustrés. En síntesis, podría decirse que el primero puede ser fuente de un desequilibrio 
emocional, cognitivo o conductual, así como de alteraciones psicosomáticas; el eustrés, en cambio, se asocia con el bienestar físico, mental y emocional. Lo más importante es lograr un nivel moderado de estrés, que permita enfrentar y resolver de forma adecuada las situaciones de la vida diaria.

Es importante que la persona conozca su umbral óptimo de estrés, de modo que no sobrepase este por un tiempo prolongado, por cuanto corre el riesgo de llegar a la tercera fase del estrés, el agotamiento.

Las respuestas al estrés son múltiples y variadas, incluyendo las físicas, las cognitivas, las emotivas y las conductuales.

Se considera la ansiedad como una de las manifestaciones psicológicas más peligrosas del estrés y a la depresión como uno de los riesgos terminales de este. De igual forma se presentan las enfermedades psicosomáticas, que se manifiestan orgánicamente pero cuyo origen es psicológico.

Igual que las respuestas a él, las causas del estrés son muchas y variadas; no obstante, pueden ser agrupadas en dos grandes fuentes: la externa proveniente de circunstancias tales como el ambiente social, la familia, el trabajo, el estudio, las relaciones interpersonales, experiencias traumáticas derivadas de catástrofes naturales y distintas molestias cotidianas; la interna está relacionada con las características de personalidad y la forma en que la persona percibe y enfrenta las situaciones de su vida.

Existen diferentes formas de prevenir el estrés, entre estas pueden citarse el fortalecimiento físico y psicológico, desarrollar actitudes más positivas y realistas frente a las situaciones de la vida, practicar ejercicio físico, cuidar el tipo de alimentación, lograr un reposo adecuado, disfrutar del contacto con la naturaleza y del tiempo libre, expresar sentimientos y pensamientos y aprender a planificar el tiempo, las actividades y los propósitos existenciales.

También, existen diversas formas de afrontar el estrés; por ejemplo, practicar ejercicios de relajación y meditación que favorezcan el logro de la tranquilidad mental y la distensión muscular, aprender a preocuparse constructivamente y sobre todo tratardedesarrollarunaactividad cognoscitiva funcional que conlleve a pensamientos racionales y por ende a emociones y conductas más apropiadas, así como a un funcionamiento fisiológico más sano.

En cuanto al ambiente educativo, puede decirse que son muchas las fuentes de estrés que pueden afectar a la población estudiantil: la competitividad, las dificultades en el rendimiento académico, los problemas en las relaciones interpersonales, la violencia física y psicológica, los temores asociados con la realización de pruebas, el fracaso y el incumplimiento de expectativas de progenitores y docentes, para mencionar algunas. Todas estas situaciones además de generar estrés pueden provocar frustración, desmotivación y ansiedad, lo cual tiene un impacto emocional, cognitivo, conductual y fisiológico negativo en el estudiantado, que repercute de la misma forma en la eficiencia de su aprendizaje y en los logros en su rendimiento académico.

Tomando en cuenta las posibles fuentes de estrés que afectan a estudiantes en las instituciones educativas y conscientes de las implicaciones negativas que tiene tanto en la vida personal como académica, debería hacerse un esfuerzo por desarrollar programas para la prevención y el afrontamiento adecuado del estrés y de sus manifestaciones más peligrosas: la ansiedad y la depresión.

El aporte que puede brindar la disciplina de la Orientación sobre estos temas es de enorme valor y conveniencia, por cuanto las personas profesionales de este campo, además de contar con la preparación necesaria para trabajar con estos, se encuentran, en muchos casos, desempeñándose en el sistema educativo.

Entre los aspectos que podrían ser tratados directamente con el estudiantado están:

El conocimiento de sí mismas o de sí mismos, de sus habilidades, debilidades, intereses y necesidades. 
Puede ser útil el tema de las inteligencias múltiples. También el conocer sus estilos personales de aprendizaje y razonamiento y el empleo, por consiguiente, de técnicas apropiadas de estudio.

Cómo lograr una mejor administración del tiempo y una organización adecuada del lugar de estudio para obtener mayor comodidad y evitar distracciones.

La importancia de tomar tiempo para la recreación, de preferencia al aire libre y en contacto con la naturaleza. De realizar otras actividades que les ayuden a renovarse física y psicológicamente, tales como practicar un deporte y realizar ejercicios de relajación. La conveniencia de mantener una dieta saludable y lograr un descanso apropiado.

De igual forma deberían tratarse temas como el establecimiento de relaciones humanas positivas mediante el empleo de la conducta y la comunicación asertivas. Asimismo, utilizando la inteligencia emocional, aprender las formas adecuadas de expresar las emociones y los pensamientos,

Enseñar al estudiantado cómo eliminar o modificar ideas irracionales respecto de sí misma o de sí mismo, de las otras personas y de diversas situaciones, de manera que su forma de pensar sea más racional o positiva, pueda experimentar emociones más saludables y desarrollar conductas más adecuadas, lo cual favorecerá su autovaloración y por ende su autoestima.

Enseñar a las personas estudiantes cómoanticiparsituacionesproductoras de estrés y cómo prepararse para afrontarlas de manera adecuada empleando técnicas de relajación, de respiración, fantasías dirigidas y el uso de auto aserciones (preparación de mensajes de enfrentamiento adaptativo que la persona debe repetir antes, durante y después de situaciones generadoras de estrés o ansiedad) para mencionar algunas.

En cuanto a las instituciones educativas, estas deberían esforzarse por crear ambientes emocionales y sociales que contribuyan a disminuir el estrés de los estudiantes; por ejemplo, equilibrando los niveles de exigencia académica y evitando la sobrecarga de trabajos extra clase que consumen todo el tiempo y no dan espacio para la recreación y el descanso. Cambiar las prácticas de competitividad por otras en las que se fomente la solidaridad entre pares trabajando de forma conjunta por un bien común. Propiciar relaciones asertivas y ayudar al estudiantado a eliminar temores sobre ciertas materias, la participación en clase y la presentación de pruebas.

Las instituciones educativas también deberían fomentar la práctica de actividades de expresión artística que permitan a la población estudiantil manifestar sus capacidades creativas de forma libre, sin competencia, comparación o calificación. Igualmente, permitir que la persona pueda descubrir su propio yo mediante el arte, canalizar y organizar sus emociones, pensamientos, percepciones y desarrollar su imaginación. Así como lo menciono en mi libro sobre estrategias para la orientación de la niñez (Naranjo, 2004b):

desarrollar una imagen positiva de sí misma o de sí mismo, aumentar la confianza en la capacidad de la autoexpresión, facilitar la oportunidad para el pensamiento divergente, deben ser los propósitos fundamentales de un programa educativo y el arte proporciona un lugar, además, para la expresión emocional, impulsando una mayor participación de lo afectivo, lo cual debe hacerse en un ambiente saludable; esto es, libre de tensiones (p. 4).

Con respecto a la familia, el estrés en los estudiantes puede ser aumentado por la dinámica familiar que se genera durante ciertos períodos; por ejemplo, en las semanas de exámenes, durante las cuales se desarrolla un ambiente desagradable, de tensión emocional, en el cual las 
relaciones y la comunicación se centran casi exclusivamente en el rendimiento académico, y de esta forma se descuidan otras áreas de vida importantes como compartir experiencias, la expresión de afecto y la recreación.

Se debe analizar con las madres y los padres de familia la importancia de mantener expectativas realistas respecto de los logros académicos de sus hijas e hijos para evitar las exigencias de perfección y la inflexibilidad, pues esto sólo conduce a la frustración, a una disminución de la autoestima y a relaciones cargadas de resentimiento o culpa, lo que finalmente no beneficia sino que perjudica el rendimiento académico de la persona y, lo más grave, daña los vínculos afectivos de la familia.

\section{Referencias bibliográficas}

Arellano, N. (2002). Estrés. Consultado el 22 de marzo de 2007, de: http://www. quadernsddigitals.net/datos-web/ emeroteca/nr_507/a-7061/7061.html.

Bensabat, S. (1987). Stress. Grandes especialistas responden. Bilbao: Mensajero.

Dumont, M., LeClerc, D., y Deslandes, R. (2003). Personal resources and psychological distress in association with the school performance and stress of fourth secondary students [Los recursos personales y los trastornos psicológicos en asociación con el rendimiento escolar y el estrés de estudiantes de cuarto año de secundaria]. Canadian Journal of Behavioral Science, 35(4), 254-267.

Ellis, A., y Abrahams, E. (1980). Terapia racional emotiva. Mejor salud y superación persona afrontando nuestra realidad. México: Pax.

Feixas, G.,yMiró, M.(1993).Aproximaciones a la psicoterapia. Una introducción a los tratamientos psicológicos. Barcelona: Paidós.

Folkman, S., Lazarus, R., Gruen, R., y De Longis, A. (1986). Appraisal, coping, health status and psychological symptoms [Valoración, afrontamiento, nivel de salud y síntomas psicológicos]. Journal of Personality and Social Psychology, 50(3), 571-579.

Garrido, M., y García, J. (1994). Psicoterapia. Modelos contemporáneos y aplicaciones. Valencia: Promolibro.

Hays, R., y Oxley, D. (1996). Social network development and functioning during a life transition [El desarrollo de redes sociales y el funcionamiento durante una transición de la vida]. Journal of Personality and Social Psychology, 49(2), 357-369.

Holahan, C., y Moss, R. (1986). Personality, coping and family resources in stress resistance. A longitudinal analysis [Personalidad, afrontamiento y recursos familiares en la resistencia al estrés. Un análisis longitudinal]. Journal of Personality and Social Psychology, 51(2), 349-395.

Jadue, G. (2001). Estudios pedagógicos. Consultado el 14 de abril de 2007, de: http://www.scielo.php?pid=507180705200100010008 script $=$ sci-art text.

Lazarus, R., y Folkman, S. (1986). Estrés y procesos cognitivos. Barcelona: Martínez Roca.

Martínez, E., y Díaz, D. (2007). Una aproximación psicosocial al estrés escolar. Educación y Educadores, 10(2). Consultado el 20 de agosto de 2008, de:http://educacionyeducadores. unisabana.edu.co/index.php/eye/ article/view/687 
Melgosa, J. (1995). Nuevo estilo de vida. ¡Sin estrés! Madrid: Safeliz.

Morris, C. (1997). Psicología. México: Prentice Hall.

Naranjo, M. L. (2004a). Enfoques conductistas, cognitivos y racional emotivos. San José, Costa Rica: Universidad de Costa Rica.

Naranjo, M. L. (2004b). Estrategias para la orientación de la niñez. San José, Costa Rica: Universidad de Costa Rica.

Neidhardt, J., Weinstein, M., y Conry, R. (1989). Seis programas para prevenir y controlar el estrés. Madrid: Deusto.
Newcomer, P. (1993). Cómo enseñar a los niños perturbados. México: FEC.

Oblitas, L. (2004). Psicología de la salud y calidad de vida. Australia: International Thompson Editores.

Rice, P. (2000). Adolescencia. Desarrollo, relaciones y cultura. Madrid: Prentice Hall.

Santrock, J. (2002). Psicología de la educación. México: McGraw-Hill.

Universidad de Sevilla. (2005). Programa Golden5: Una intervención psicoeducativa. Consultado el 14 de abril de 2007, de: http://www.golden5.org/ golden5/ 